\title{
Consensus and Discordance in the Management of Growth Hormone-Treated Patients: Results of a Knowledge, Attitudes, Beliefs, and Practices Survey
}

\author{
Bradley S. Miller, ${ }^{1}$ Dorothy I. Shulman, ${ }^{2}$ Alicia Shillington, ${ }^{3}$ Qing Harshaw, ${ }^{3}$ \\ Darrell M. Wilson, ${ }^{4}$ David Schwartz, ${ }^{5}$ Michael Kappy, ${ }^{6}$ Bert Bakker, ${ }^{7}$ and David Wyatt ${ }^{8}$ \\ ${ }^{1}$ Department of Pediatrics, University of Minnesota Amplatz Children's Hospital, MMC 8404, PWB 13-124, \\ 516 Delaware Street, SE, Minneapolis, MN 55455, USA \\ ${ }^{2}$ All Children's Hospital, University of South Florida, Tampa, FL 33701, USA \\ ${ }^{3}$ EPI-Q, Oakbrook, IL 60523, USA \\ ${ }^{4}$ Lucile Packard Children's Hospital, Stanford University, Palo Alto, CA 94304, USA \\ ${ }^{5}$ Department of Pediatrics, University of South Carolina, Columbia, SC 29208, USA \\ ${ }^{6}$ Children's Hospital, University of Colorado, Denver, CO 80218, USA \\ ${ }^{7}$ Genentech, Inc., South San Francisco, CA 94080, USA \\ ${ }^{8}$ Children's Hospital, Medical College of Wisconsin, Milwaukee, WI 53226, USA
}

Correspondence should be addressed to Bradley S. Miller, mille685@umn.edu

Received 21 April 2010; Revised 24 July 2010; Accepted 2 August 2010

Academic Editor: Allen W. Root

Copyright (C) 2010 Bradley S. Miller et al. This is an open access article distributed under the Creative Commons Attribution License, which permits unrestricted use, distribution, and reproduction in any medium, provided the original work is properly cited.

\begin{abstract}
Our purpose was to determine pediatric endocrinologists' knowledge, attitudes, beliefs, and practices (KABPs) regarding recombinant human growth hormone (rhGH) treatment, examine care-related attitude consensus or discordance, and identify evidence-based practice gaps. We developed a survey for National Cooperative Growth Study (NCGS) investigators $(N=711)$ to elicit their KABPs regarding GH stimulation testing as a diagnostic tool, IGF-1 monitoring for safety and dosing guidance, and pubertal dosing. Responses were compared with NCGS data from the last 20 years. Comparison between survey responses and NCGS data revealed potential discrepancies between expressed opinions and actual practice. In conclusion, this KABP survey, combined with NCGS data, suggests changes over time in diagnostic and rhGH-related therapeutic practices. Variability and inconsistency exist between the survey responses and practice trends over time as reflected in the NCGS database. Further study is necessary to provide evidence to guide rhGH treatment decisions.
\end{abstract}

\section{Introduction}

Therapy with growth hormone, initially derived from cadaveric human pituitary glands, began in the late 1950s and expanded with the availability of recombinant human growth hormone (rhGH) in1985. rhGH therapy for growth disorders in children and, more recently, for replacement or anabolic actions in adults, has been prescribed and monitored primarily by endocrinologists and nephrologists. Prescribing and monitoring practices have evolved over time based upon the availability of rhGH, hormonal assays, and experience in the treatment of an expanding list of indications. However, the ongoing debates regarding diagnostic and treatment paradigms, and the changing practice guidelines and their interpretation by third-party payers, emphasize the lack of extensive evidence for our current practices. In order to improve the care of children with growth disorders, we sought to identify specific areas in which evidence suggests a need for further study and in which there is great variability in clinical practice. The objectives of our efforts were to determine attitudes and beliefs of pediatric endocrinologists regarding treatment of children receiving rhGH, examine areas of consensus and discordance in attitudes related to care delivery, 
and identify gaps in evidence-based practice for quality improvement. In order to achieve this goal, the Knowledge Attitudes Beliefs Practice (KABP) process was utilized. First, an analysis of the literature regarding GH treatment of children and adolescents using methods described by the Cochrane collaboration was performed to assess existing evidence-based practice recommendations and treatment guidelines (Knowledge) [1]. We then developed a survey based upon existing evidence gaps and surveyed investigators participating in the phase 4 postmarketing safety surveillance trial, the National Cooperative Growth Study (NCGS), to determine current opinions regarding treatment processes (Attitudes and Beliefs). In order to evaluate how Attitudes and Beliefs are translated into clinical practice, we compared the survey responses to actual practice based upon data entered into the NCGS registry (Practice).

The NCGS database has enrolled 55,000 children treated with Genentech rhGH products in North America between the years 1987 and 2005. The purpose of the database was to gather safety and efficacy data on the use of rhGH in the postmarketing setting. Because of the large number of children in the database, the relative risk of rare side effects can be estimated. In addition, the size of the study provides sufficient patient information to aid in determining the baseline and treatment factors that impact the growth response in rhGH-treated children.

\section{Methods}

To develop the survey content, we reviewed the most recent guidelines from the American Association of Clinical Endocrinologists (AACE) [2], the Endocrine Society (ES) [3], the Growth Hormone Research Society (GHRS) [4], and the Lawson Wilkins Pediatric Endocrine Society (LWPES) [5] and extracted specific recommendations for diagnosis and treatment. In addition, a Medline search was conducted on the terms "growth hormone," "growth hormone deficiency," "Turner syndrome," "craniopharyngioma," and "Prader-Willi syndrome." The search was limited to papers published since the most recent updates of the guidelines (2003-2006). This search yielded 387 citations. The search was then limited further to those papers that included aspects of care related to diagnosis of growth hormone deficiency (GHD), growth hormone treatment initiation, dosing, monitoring, and duration of therapy. This yielded 149 articles. Articles were reviewed by teams of two, including a clinician and an epidemiologist, using criteria established by the Cochrane collaboration [1]. Articles were ranked on strength of evidence and whether or not the conclusions supported either a new or existing aspect of care as recommended by guidelines [6].

Simultaneously, a panel of six academic pediatric endocrinologists with special expertise in growth disorders was recruited by members of the NCGS investigators' advisory board. Results of the literature review were summarized and disseminated to the panel, and a meeting was convened in September 2006 to formulate the survey content. A modified RAND [7] approach was used to identify and rank the relative importance of each aspect of care to be included on the survey. Statements were developed regarding care, and respondents were asked to rank their level of agreement or disagreement with each statement. The following is a sample question: "If it were not required for reimbursement, I would continue to perform growth hormone stimulation testing on my patients that I am evaluating for GHD." The respondent was asked to circle a number ranging from 1 through 7, with 1 representing "strongly agree," 4 representing "agree," and 7 representing "strongly disagree." Other questions necessitated picking a single answer or ranking responses. The final survey included questions on screening studies performed in short children, criteria for screening, diagnosis of GHD, rhGH dosing, monitoring, assessing growth response, determining when to stop therapy, and reevaluating at adult height.

Surveys were mailed to all active principal investigators and subinvestigators who had patients enrolled in the NCGS registry $(N=711)$. Respondents were given return postagepaid envelopes and asked to complete the surveys over a 6week period. Results were tabulated and data were analyzed using SPSS 13.0 (Chicago, IL). For questions requiring agreement or disagreement percentage, respondents answering with a value of 1 to 4 were considered to be in agreement, and those responding with a value of greater than 4 were considered to be in disagreement.

To facilitate comparison between survey data and clinical practice, data were abstracted from the NCGS database. Following obtainment of informed consent/assent, data for rhGH-treated children are entered anonymously into the database. Required data for initial enrollment include date of birth, sex, pretreatment height and weight, pubertal staging, diagnosis (according to investigator), rhGH dosage, and start date of therapy. Required data from subsequent clinic visits include date of visit, height, weight, pubertal staging, and dosage prescribed at the visit. Optional data such as parental heights, birth weight, previous growth measurements, concomitant medications, bone age results, insulin-like growth factor 1 (IGF-1) levels, thyroid function, and results of growth hormone or other pituitary testing may also be entered. For the purposes of this analysis, data were analyzed from the time that the NCGS was rolled out to most major endocrine sites (1987), and for the most recent 5-year period.

Statistical analyses were performed at the 5\% significance level using two-sided tests. Quantitative variables are summarized using means, standard deviation scores (SDS), and Student's $t$-test where appropriate. Qualitative variables are summarized using number of nonmissing data, counts, percentages, and $\chi 2$ test where appropriate.

\section{Results}

3.1. Strength of Evidence in Literature. Articles $(n=140)$ were ranked on strength of evidence and whether or not the conclusions supported either a new or existing aspect of care as recommended by guidelines (Table 1) $[1,6]$. 
TABLE 1: Characteristics of articles reviewed.

\begin{tabular}{lc}
\hline & $N=149$ \\
\hline Evidence Type & $\%$ \\
Randomized controlled trials (large) & 5 \\
Randomized controlled trials (small) & 21 \\
Nonrandomized trials & 13 \\
Case studies/series & 19 \\
Observational studies & 39 \\
Expert opinion & 3 \\
\hline Evidence Strength* & $\%$ \\
Level 1 (a-c) & 5 \\
Level 2 (a-c) & 27 \\
Level 3 (a-b) & 24 \\
Level 4 & 39 \\
Level 5 & 5 \\
\hline
\end{tabular}

* Level 1 (a-c): systematic reviews, large randomized controlled trials; Level 2 (a-c): small or underpowered clinical trials, cohort studies; Level 3 (a-b): case control studies, other epidemiology or outcomes studies; Level 4: case series; Level 5: expert opinion. Articles reviewed available in Supplemental Reference List.

TABLE 2: Characteristics of survey respondents.

\begin{tabular}{lc}
\hline & $N=207$ \\
\hline $\begin{array}{l}\text { Age (mean, range) } \\
\text { Gender }\end{array}$ & $52(31-77)$ \\
$\quad$ Male, \% & \\
Specialty & 95 \\
$\quad$ Pediatric endocrinology, \% & 2 \\
$\quad$ Nephrology, \% & 3 \\
Other, \% & 37 \\
Private practice, \% & 91 \\
Teaching medical students, \% & 88 \\
Board certified, \% & \\
Years in practice & 11 \\
0-5 years, \% & 12 \\
6-10 years, \% & 19 \\
11-15 years, \% & 15 \\
16-20 years, \% & 43 \\
> 20 years, \% & $17(3-40)$ \\
Years prescribing growth hormone-mean (range) & $95(8-500)$ \\
Current number of treated patients-mean (range) &
\end{tabular}

3.2. Demographics. Of 711 surveys mailed to NCGS investigators, 207 (29\%) were returned by the deadline for analysis. A total of 260 surveys were eventually returned (37\% response rate). This response rate is comparable to a 2007 survey of LWPES members used to guide organizational priorities (33\%, personal communication LWPES office). Respondents were primarily board-certified pediatric endocrinologists (Table 2) involved in teaching medical students. The mean age of respondents was 52 years, with a significant proportion $(43 \%)$ in practice for more than 20 years.
3.3. Screening the Poorly Growing Child. Most clinicians strongly agreed that an initial evaluation should occur at height $\leq-2.0$ SDS $(82 \%)$ or $\geq 1.5$ SDS below midparental height SDS (87\%). Review of entry height for children in the NCGS database treated with rhGH for all etiologies of short stature shows that $75 \%$ had an initial height $\leq-2.0$ SDS, with a mean entry height of -2.6 SDS.

With regard to initial laboratory evaluation in the poorly growing child, 95\% of respondents endorsed obtaining a bone age radiograph and thyroid functions; $94 \%$, serum IGF-1 levels; 91\%, chemistry panel; 87\%, a karyotype in females. A complete blood count, IGF-binding protein 3 (IGFBP-3), urinalysis, sedimentation rate, and screening for celiac disease were included by $75 \%$ to $85 \%$ of respondents. Analysis of the NCGS data reveals that a bone age radiograph $(76 \%)$ is the most common screening test recorded for all children entered in the study. IGF-1 (24\%) and IGFBP-3 $(16 \%)$ are the next most common screening tests recorded.

3.4. Diagnosis of GHD. Seventy percent of all children entered into NCGS since 1987 are recorded as having undergone growth hormone stimulation testing (GHST). This fraction has been steadily decreasing over time, with only $53 \%$ recorded as having undergone GHST during the period from 2000 to 2005 (Table 3). Even in children diagnosed with organic GHD, the rate of GHST recorded has declined from 74\% overall to 49\% from 2000 to 2005 . GHST use in children with Turner syndrome and children diagnosed as small for gestational age without catch-up growth has declined to minimal use (Table 3 ).

According to the survey, $18 \%$ of clinicians continue to routinely use the insulin tolerance test (ITT). The recorded use of the ITT in NCGS has also declined over time (29\% of all patients since 1987; 20\% between 2000 and 2005).

Respondents felt that GHST was not necessary prior to initiating rhGH therapy in a poorly growing child with documentation of either a structural pituitary abnormality or a known hypothalamic pituitary insult (i.e., radiation, surgery, or trauma; $21 \%$ ), low serum IGF-1 (for age, gender, and sexual maturation; $2 \%$ ), or both (33\%). Thus, a total of $56 \%$ of respondents felt there were circumstances in which GSHT was not necessary prior to rhGH therapy. Twenty-nine percent felt that GHST should always be performed when considering the diagnosis of GHD. Survey respondents who had been practicing pediatric endocrinology for 16 or more years or longer were significantly more likely to report that GHST should always be performed than those who had been practicing for 15 or fewer years $(37 \%$ versus $21 \%, P<.05)$.

When asked what criteria would be necessary to make a diagnosis of GHD if GHST was not required by payers, $57 \%$ felt that GHST was necessary to make a diagnosis of GHD, and 55\% reported they would continue to perform GHST. Despite this response, low serum IGF-1, low growth velocity, magnetic resonance imaging (MRI) evidence, low IGFBP-3, and low height were all ranked higher than GHST as requirements for making a diagnosis of GHD (Figure 1).

Eighty-two percent answered that a low level of serum IGF-1, in the absence of malnutrition and chronic illness, 
TABLE 3: Use of GHST and IGF-1 testing reported in the NCGS registry.

\begin{tabular}{lcc}
\hline & All years $N=51,909$ & $2000-2005 n=16,481$ \\
\hline Stimulation test performed, \% & 70 & 53 \\
Stimulation testing only performed, \% & 50 & 18 \\
Stimulation testing performed with IGF-1, \% & 20 & 35 \\
IGF-1 test performed, \% & 24 & 41 \\
IGF-1 test performed in absence of stimulation testing, \% & 4 & 6 \\
Stimulation test performed with insulin, \% & 29 & 20
\end{tabular}

GHST: growth hormone stimulation test; IGF-1: insulin-like growth factor 1; NCGS: National Cooperative Growth Study.

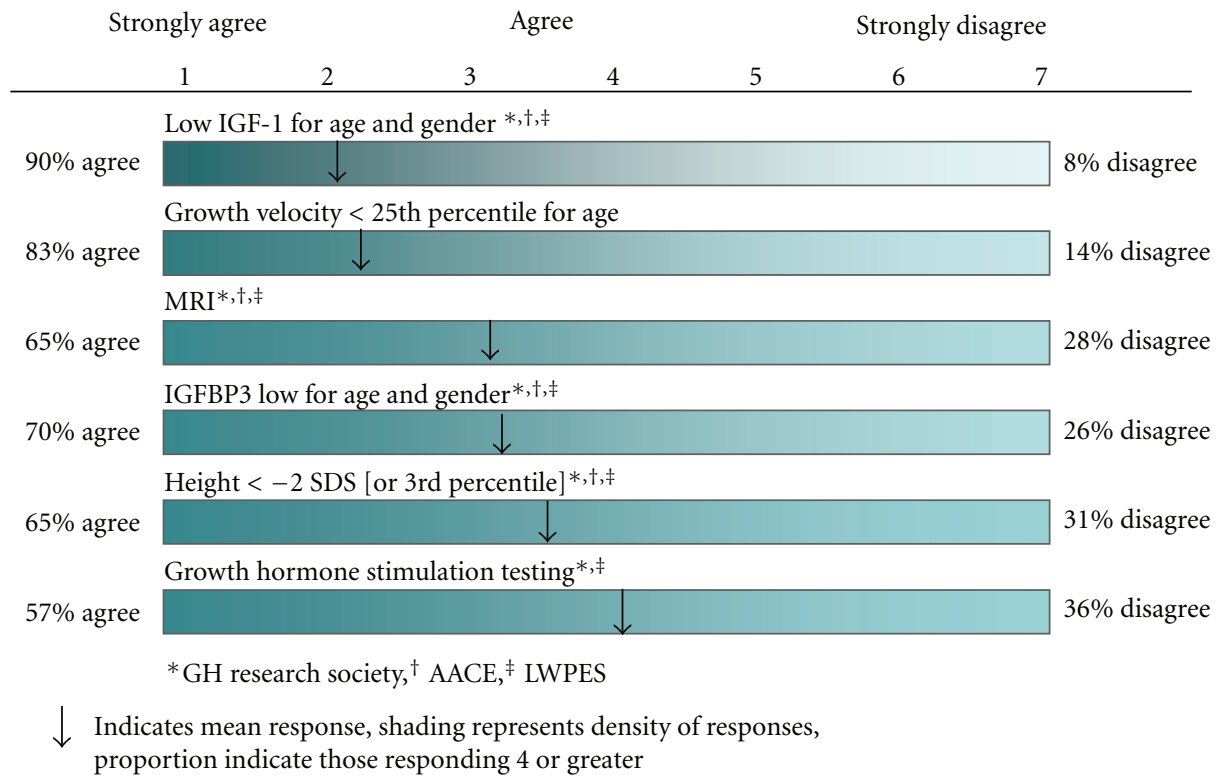

FIGURE 1: Responses to the survey question: "Assuming stimulation testing is not required by payers, the following are necessary to make a diagnosis of GHD."

was equivalent to $(45 \%)$ or better than $(37 \%)$ GHST. Those in nonteaching settings were significantly more likely than those in teaching settings to have this opinion (100\% versus $82 \%, P<.05)$. There was no statistical difference based upon years in practice. Diagnostic measurement (and recording in NCGS) of serum IGF-1 in children with short stature of all etiologies has increased over time (24\% all years, 41\% 20002005 ), but its use as an exclusive diagnostic tool in GHD is seen only in a small number of patients in the database $(4 \%$ all years, 6\% 2000-2005; Table 3).

3.5. Monitoring Serum IGF-1 During rhGH Therapy. Routine measurement of serum IGF-1 levels has been proposed for both safety and efficacy monitoring [8]. In our survey, respondents endorsed measuring serum IGF-1 routinely $(72 \%)$ and making rhGH dose adjustments to maintain the IGF-1 in the midnormal $(66 \%)$ or high-normal $(55 \%)$ range. However, more common reasons for dose adjustment were growth response (85\%) and weight (80\%). In addition, $22 \%$ would measure serum IGF-1 only when patients were responding poorly. In patients with elevated serum IGF-1, $55 \%$ reported that they would decrease the rhGH dose, $28 \%$ would make no change, and 15\% reported that they do not use serum IGF-1 to adjust therapy. Analysis of NCGS data suggests that in actual practice only $41 \%$ of children have an IGF-1 recorded on at least an annual basis.

3.6. Assessing Response to rhGH Therapy. Eighty-seven percent of the survey participants responded that they would always determine growth velocity prior to initiation of rhGH therapy. However, pretreatment growth velocity was recorded for only $48 \%$ of children entered into the NCGS database. When asked what constituted a poor response to rhGH therapy, the least stringent and most easily measured minimal response, an increase of $<2 \mathrm{~cm} /$ year over baseline, was most popular $(85 \%)$ among respondents. Slightly fewer indicated that an increase of $<0.25$ height SDS/yr $(78 \%)$ and $<50 \%$ increase in growth velocity over baseline $(77 \%)$ were reasonable criteria. When asked what the duration of an adequate trial of rhGH therapy should be, most considered assessing adequacy of growth response after 3 $(40 \%)$ or $6(50 \%)$ months of therapy. This reported practice is supported by the first documented entry of treatment response data into NCGS at $3(65 \%)$ and 6 (20\%) months.

If the growth response to $\mathrm{rhGH}$ therapy is deemed inadequate in a patient, most $(86 \%)$ responded that they 
would use serum IGF-1 levels to guide their decision whether to increase the rhGH dose or stop therapy. Many (69\%) would increase the rhGH dose. Most (66\%) disagreed with stopping treatment based upon a poor growth response alone.

To compare these reported decision points to practice, we analyzed the first-year growth response of children with GHD or idiopathic short stature (ISS, short stature with no evidence of illness, and normal GHST) in the NCGS database. Among those who had a recorded pretreatment growth velocity, $11 \%$ of GHD patients and $12 \%$ of ISS patients had a first-year increase of $<2 \mathrm{~cm} / \mathrm{yr}, 13 \%$ of both groups had an increase of $<0.25$ height SDS/yr, and $16 \%$ of both groups had $<50 \%$ increase in growth velocity. More than $40 \%$ of children who had a poor growth response, as defined by any of the three criteria listed above, were continued on the same dose of rhGH therapy. This was the case for children with GHD whether or not a serum IGF1 was recorded. However, if an IGF-1 level was recorded for children with ISS, the dose of rhGH was more likely to be increased $(64 \%)$ than for children in whom it was not recorded $(47 \%, P<.05)$.

3.7. Pubertal Dosing. To assess the impact of the study of Mauras et al. [9], which reported the results of an increase in the dose of rhGH during puberty to $0.7 \mathrm{mg} / \mathrm{kg} /$ week, a dose that was subsequently approved by the U.S. Food and Drug Administration (FDA) in 2000, we asked if survey respondents felt that rhGH dosing should be increased in all pubertal patients with GHD (35\% agreed) or if the increase should be reserved for those who are not expected to reach target height (59\% agreed). The NCGS registry data support the survey results: $37 \%$ of patients received rhGH doses $>0.4 \mathrm{mg} / \mathrm{kg} /$ wk during puberty (Tanner stages $2-4)$. Only $10 \%$ were prescribed the $0.7 \mathrm{mg} / \mathrm{kg} /$ week dose. The degree of short stature did not appear to impact the decision, as frequency of pubertal dosing was not increased in children whose height was $<-2$ SDS. Similar percentages of respondents $(56 \%)$ felt that pubertal dosing should be used in pubertal-age children with ISS who are not expected to reach target height. Twelve percent stated that pubertal dosing should rarely, if ever, be used in ISS, whereas no respondents expressed this opinion for the diagnosis of GHD.

3.8. End of rhGH Therapy. Surveyed physicians were asked to rank factors that were considered important in the decision to end rhGH therapy. Documentation of epiphyseal closure was ranked first by $39 \%$. Continuation of rhGH treatment until the patient was comfortable with height and slowed growth velocity to $<2 \mathrm{~cm} / \mathrm{yr}$ were ranked first by $23 \%$ and $21 \%$ of respondents, respectively, and had an identical rank mean score (3.0). In the NCGS database, an explanation for discontinuation of rhGH was provided for only $7 \%$ of children; of this small group, $86 \%$ were still growing at least $2 \mathrm{~cm} / \mathrm{yr}, 82 \%$ were within 2 SDS of target height, $60 \%$ were $>5$ th percentile for adult height, and only $13 \%$ were near epiphyseal closure (bone age $>15$ years in girls, $>17$ years in boys) (Table 4 ).

\subsection{Treatment into Adulthood for the GHD Adolescent.} GHD in adults is an indication for rhGH therapy. Figure 2 illustrates the response to a question regarding cessation of therapy and the need for diagnostic retesting. When asked if rhGH should be stopped in adolescents with idiopathic GHD when growth is complete, $74 \%$ respondents indicated that they would stop treatment. Eighty-four percent agreed with continuing therapy if repeaingt GHST reveals a peak $\mathrm{GH}$ response of $<5 \mathrm{ng} / \mathrm{mL}$. Fifty-five percent would continue therapy based upon a low serum IGF-1 level alone (following temporary cessation of GH treatment). In children with multiple pituitary hormone deficiencies, $84 \%$ agreed that rhGH treatment should continue without interruption.

Table 5 summarizes the comparison of the published clinical practice guidelines, survey responses, and NCGS registry data for each clinical question.

\section{Discussion}

This study uses the KABP process to identify current gaps in the evidence for clinical care in rhGH therapy (Knowledge), determine the opinions of a group of rhGH prescribers (Attitudes and Beliefs), and compare the opinions reflected by that survey to data entered into an rhGH safety and efficacy registry (NCGS) with voluntary data fields (Practice). The goal was to identify specific areas of clinical practice variability that may identify areas of consensus and disagreement where evidence-based data are lacking to provide a basis for future knowledge development which could improve care of children treated with rhGH.

Respondents to the survey, 29\% of NCGS investigators, were largely experienced, board-certified endocrinologists who held teaching positions and were members of the LWPES. Although this is a low survey response rate, a similar 2007 survey of LWPES members yielded a similar response rate. In addition, the demographics of our survey were similar to the 2007 LWPES survey except that our study had a slightly higher rate of responders in private practice ( 37 versus $24 \%$ ), fewer responders in practice $>20$ years ( 43 versus $55 \%)$, and more responders in practice $<5$ years $(11 \%$ versus $4 \%$ ). However, regardless of the demographics, due to the low response rate, the responses described may not accurately reflect the opinions of all NCGS investigators or the larger pediatric endocrinology community.

The use of the NCGS database to reflect current and previous practice may not reflect practice in all children receiving rhGH during this period. Estimates based upon the number of children receiving rhGH products eligible for enrollment in the study who have data entered and the market share of these products suggest that NCGS data reflect approximately $30 \%$ of nearly 200,000 children treated with rhGH since its approval in 1985. In addition, NCGS data may not be a complete and accurate reflection of practice in those children entered into the database due to the absence of potentially relevant data. However, the NCGS 


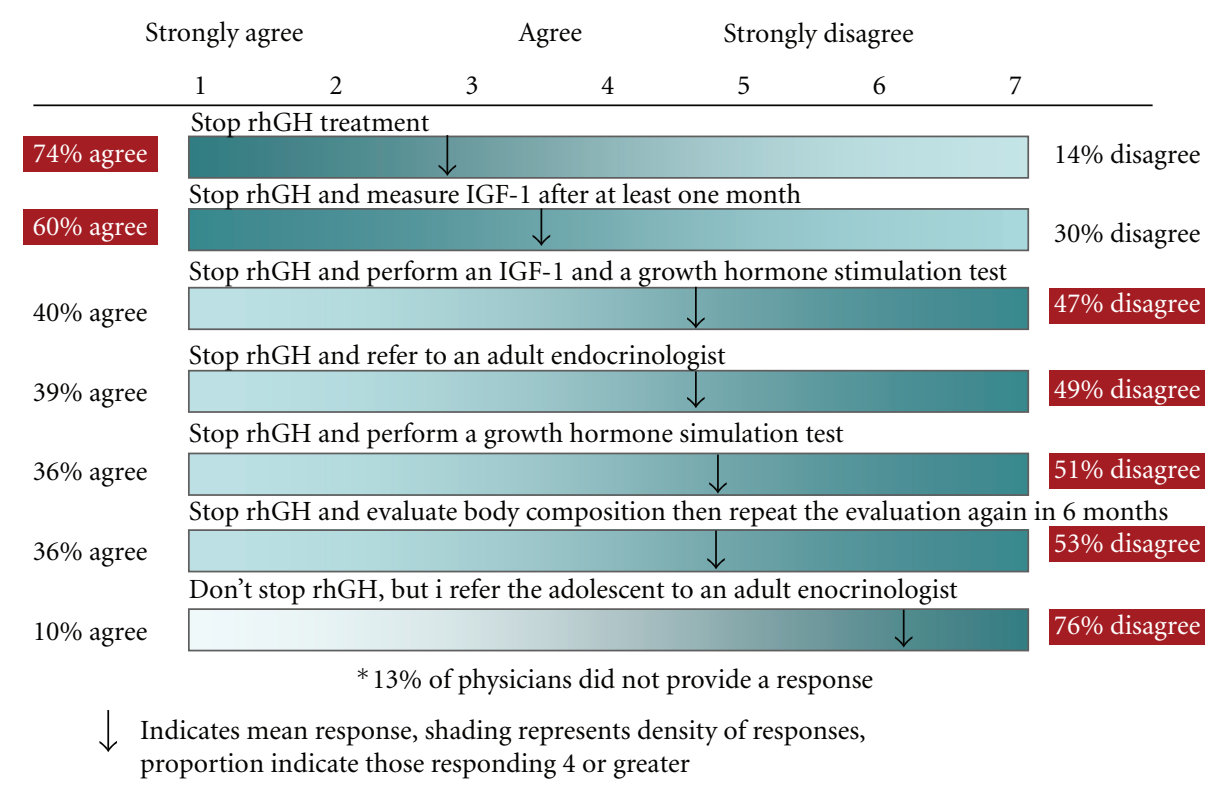

FIGURE 2: Responses to the survey question: "In idiopathic isolated GHD adolescents who are at the end of linear growth, I generally...."

TABLE 4: Factors influencing decision to discontinue a course of rhGH.

\begin{tabular}{lcc}
\hline & Rank (mean score) $N=207$ & Proportion ranked as \#1 $N=207$ \\
\hline Epiphyseal closure is documented & $1(2.5)$ & $39 \%$ \\
Patient is comfortable with his/her height & $2 *(3.0)$ & $23 \%$ \\
Patient's growth velocity is $<2 \mathrm{~cm} / \mathrm{yr}$ & $2 *(3.0)$ & $21 \%$ \\
Patient's growth velocity is $<1 \mathrm{~cm} / \mathrm{yr}$ & $4(3.1)$ & $12 \%$ \\
Patient is at least at the 5 th percentile for adult population & $5(4.6)$ & $4 \%$ \\
Patient is within two SDs of target height & $6(4.7)$ & $2 \%$ \\
\hline
\end{tabular}

rhGH: recombinant human growth hormone; SD: standard deviation.

*Indicates a tie.

is the largest data repository for rhGH therapy in children in the U S Furthermore, measures of practice may not reflect attitudes, beliefs, and opinions due to insurance requirements.

\subsection{Screening Poorly Growing Children and Diagnosis of} GHD. The survey consensus was that children should be screened for GHD when height is $<-2.0$ SDS (or $>1.5$ SDS below midparental height). This consensus is consistent with current practice guidelines $[1,3,4]$ and is supported by the characteristics of children enrolled in NCGS (mean height SDS $-2.6,75 \%<-2.0 \mathrm{SDS}$ ). In addition to screening for GHD, the initial evaluation of children with short stature is targeted to identify any chronic illness that could potentially affect growth. Survey responders endorsed the following screening tests most strongly: baseline growth velocity, bone age, thyroxine, TSH, IGF-1, chemistry panel, and karyotyping for girls. In a previous study of pediatric endocrinology practices [10], these screening tests had a similar rank order of use. However, the actual frequency of these tests on the initial visit was reported to be significantly lower even in children with severe short stature (height SDS $<-3.0$ SDS and/or $<-1.5$ SDS below midparental height).
It was also observed that most children never returned for a requested second visit [10]. The latter finding emphasizes the importance of obtaining screening laboratory studies at the initial visit and/or stressing the importance of followup visits to determine the growth velocity [11].

How or whether to make a diagnosis of GHD prior to initiating therapy remains an area of controversy. Accurate diagnosis of GHD is crucial to the identification of children who are at risk of having or developing other pituitary hormone deficiencies, in particular adrenal insufficiency [12]. Current guidelines include short stature with poor growth velocity, low GH peak following GHST, low serum IGF-1 and IGFBP-3, and delayed bone age as typical features of GHD, with GHST remaining the gold standard [2-5]. The combination of poor height velocity and low IGF-1 has also been shown to correlate well with GHD [13]. However, the use of a combined approach including GHST, IGF-1, and IGFBP-3 levels improves specificity of GHD diagnosis [14]. The use of GHST, and particularly ITT, has declined over time [15]. The reasons for decreased use of GHST may include a lack of reproducibility, lack of personnel to perform the test, confounding influence of pubertal stage and body mass index, expense, and safety risk [15-17]. 
TABLE 5: Comparison of published clinical practice guidelines, survey responses, and NCGS registry data.

\begin{tabular}{|c|c|c|c|}
\hline Clinical question & Guidelines & Survey response & NCGS data \\
\hline $\begin{array}{l}\text { Height SD prompting } \\
\text { evaluation of GHD }\end{array}$ & $\begin{array}{l}<-3^{\mathrm{a}},<-2.25^{\mathrm{b}},<-2^{\mathrm{c}}<-2 \mathrm{SD} \\
\text { plus } \mathrm{GV}<-1 \mathrm{SD}^{\mathrm{a}, \mathrm{c}}>1.5 \mathrm{SD} \text { than } \\
\mathrm{MPH}^{\mathrm{a}, \mathrm{c}}>2 \mathrm{SD} \text { than } \mathrm{MPH}^{\mathrm{b}}\end{array}$ & $\begin{array}{l}<-3 \text { SD } 88 \%<-2 \text { SD } 82 \%>1.5 \\
\text { SD below MPH } 87 \%\end{array}$ & $\begin{array}{l}\text { At start of treatment }<-2 \mathrm{SD} \\
75 \%\end{array}$ \\
\hline $\begin{array}{l}\text { Is GHST necessary to } \\
\text { make diagnosis of GHD? }\end{array}$ & $\begin{array}{l}\text { GHST }<10 \text { mcg/L plus IGF-1 } \\
<-2 \text { SD important for } \\
\text { diagnosis }^{\mathrm{a}} \text {. GHST optional if } \\
\text { structural pituitary lesion, } \\
\text { surgery, radiation, or MPHD }{ }^{\mathrm{b}} \text {. }\end{array}$ & $\begin{array}{l}\text { GHST should always be } \\
\text { performed }(29 \%) \text {. } \\
\text { In presence of structural } \\
\text { abnormality or pituitary insult } \\
\text { plus low IGF-1, GHST not } \\
\text { necessary }(33 \%) \text {. }\end{array}$ & $\begin{array}{l}\text { Recorded GHST } \\
\text { All Patients 1987-2005 70\% } \\
\text { 2000-2005 53\% } \\
\text { OGHD Patients 1987-2005 74\% } \\
\text { 2000-2005 49\% }\end{array}$ \\
\hline
\end{tabular}

If not required by insurance would you Important for diagnosis of continue to perform $\mathrm{GHD}^{\mathrm{a}}$.

$55 \%$ would still perform GHST N/A GHST?

$\begin{array}{ll}\text { Value of IGF-1 in } & <-2 \text { SD required }{ }^{\mathrm{a}} . \\ \text { diagnosis of GHD } & <-2 \text { SD strongly suggestive }{ }^{\mathrm{c}} . \\ & \text { GHD unlikely if IGF-1 } 1 \geq 0 \mathrm{SD}^{\mathrm{b}} .\end{array}$

Low IGF-1 in absence of disease and malnutrition equivalent $(45 \%)$ or better $(37 \%)$ than GHST. of IGF-1.

Monitoring of IGF-1 is useful ${ }^{\mathrm{a}}$. Yearly monitoring of IGF-1 is useful $^{\mathrm{b}}$.

Routine monitoring of IGF-1 on GH therapy

If IGF-1 > +2 SD after first two years of therapy, $\mathrm{GH}$ dose reduction should be considered ${ }^{\mathrm{b}}$.

Growth response (85\%) and weight gain $(80 \%)$ were more commonly endorsed as basis of GH dose adjustments than IGF-1 targets $(55 \%-62 \%)$.

$86 \%$ endorsed use of IGF- 1 to
Height should increase $>+0.25$

SD in first yearb.

Assessing response to $\mathrm{GH}$ therapy

GH dose increase may be considered if compliance and other causes of poor growth eliminated ${ }^{\mathrm{b}}$.

$\mathrm{GH}$ at a dose of $0.7 \mathrm{mg} / \mathrm{kg} / \mathrm{wk}$ increased near adult height by 4 $\mathrm{cm}$ (0.7 SD) compared to $0.3 \mathrm{mg} / \mathrm{kg} / \mathrm{wk}$ dose $^{\mathrm{c}}$.

Repeat GHST not necessary if MPHD, severe organic GHD, or genetic defects ${ }^{\mathrm{a}, \mathrm{b}}$.

Transition evaluation Re-evaluate with GHST ${ }^{\mathrm{a}, \mathrm{b}}$ and IGF- ${ }^{\text {a }} 1-3$ months after stopping GH.
Pubertal dosing should be used: in all GHD patients (35\% agree); only in patients not expected to reach target height (59\% agree)
$72 \%$ endorse routine monitoring guide GH dose increase if growth response is poor.

Recorded IGF-11987-2005 24\%

2000-2005 41\%IGF-1 sole

diagnostic tool recorded in $4 \%-6 \%$

$41 \%$ have IGF- 1 recorded at least annually (2000-2005).

GH dose was more likely to be increased in ISS patient growing poorly in the first year of therapy if IGF-1 was measured.

No impact of IGF-1 measurement on $\mathrm{GH}$ dose increase in IGHD or OGHD poor responders.

Poor response rate $<+0.25$

Height SD (13\%)

$\mathrm{GV}$ increase of $<2 \mathrm{~cm} / \mathrm{yr}$ $(11 \%)<50 \%$ GV increase $(16 \%)$

No dose change made in $>40 \%$ of poor responders (IGHD or OGHD).

GH Dose during puberty $>0.4 \mathrm{mg} / \mathrm{kg} / \mathrm{wk}$

$37 \%>0.6 \mathrm{mg} / \mathrm{kg} / \mathrm{wk} 10 \%$

Height $<-2$ SD was not more common among those receiving these higher GH doses.

In children with MPHD GH need not be discontinued ( $84 \%$ agree); would resume $\mathrm{GH}$ with finding of low IGF-1 alone after stopping $\mathrm{GH}$ therapy for at least a month (55\% agree).

$\mathrm{GRS}^{\mathrm{a}} ; \mathrm{LWPES}^{\mathrm{b}} ; \mathrm{AACE}^{\mathrm{c}}$.

In our survey, there was general agreement that a low IGF-1 for age, gender, and pubertal status in the absence of malnutrition or chronic illness was equal to or better than GHST. However, nearly a third of respondents felt that GHST should always be performed, and more than half would continue to perform GHST in order to make a diagnosis of GHD even if not required by third-party payers. Despite some evidence impugning the validity of GHST due to a high false-positive rate, this mixture of opinions likely reflects the lack of a definitive alternative for the diagnosis of GHD. Another confounding issue is that interpretation of serum IGF-1 values remains difficult because of the lack of assay standardization and normative data for each current assay for each age and stage of puberty [18]. Currently, there is 
no single test that can consistently differentiate ISS from idiopathic GHD [19]. This continues to be an area that requires investigation.

Because of the low false-negative rate for GHST, it has been reported that children with a normal response to GHST do not require any further pituitary evaluation [19]. However, following diagnosis of GHD based upon an abnormal GHST, it has been recommended that further functional testing of pituitary function and a brain MRI be performed [2,4]. Brain MRI with focus on the pituitary has been proposed as an alternative to GHST for diagnosis of GHD [20]. Pituitary MRI has been shown to be more predictive than GHST of need for rhGH replacement in an adult [21]. In our survey, $65 \%$ of respondents indicated that an MRI was a necessary test for the diagnosis of GHD if GHST was not required.

4.2. Response to rhGH Therapy. Increased growth rate is the most commonly measured response to rhGH therapy. However, an adequate response, compared with an accurate pretreatment growth velocity, had not been defined at the time of this survey. Published consensus treatment guidelines have provided various metrics that have been adopted. Our survey respondents endorsed the use of an increase of $>2 \mathrm{~cm} / \mathrm{yr}$ over baseline growth velocity. The minimum duration of such a trial or the earliest point at which to judge the clinical response remains unclear. Because of the variability of growth and measurement error, it has been proposed that the minimum interval between growth measurements used to assess the response should be at least 6 months [22]. Recently, in an effort to identify expected growth response to rhGH therapy in the first year of therapy in children diagnosed with GHD, further analysis of NCGS data was performed to establish growth velocity response curves [23]. This paper provides reference data against which to compare a child's first-year growth velocity on treatment and which may be used to assess compliance, accuracy of diagnosis, the need for dose adjustment, consideration of concomitant illness, or discontinuation of therapy. The authors proposed that a response $>1$ SD below the mean for age and diagnosis should be considered a suboptimal response.

4.3. Monitoring Serum IGF-1 during rhGH Therapy. Although most investigators in our survey support measuring serum IGF-1 during therapy, this test was frequently not entered into the NCGS database. In addition, many investigators did not act upon the IGF-1 values obtained. This finding suggests that once rhGH therapy has been initiated, physicians are reluctant to discontinue it despite a lack of demonstrated response. A recent study demonstrated that adjusting rhGH doses to the target IGF-1 at +2 SDS regardless of growth rate led to improved growth response in the first year of therapy in some children, while others responded as well at lower IGF levels [24]. The authors concluded that some combination of growth rate and IGF targeting might be applicable. In addition, the safety of IGF targeting, which may require very high rhGH doses in some children, remains to be determined and that the use of serum IGF-1 to adjust rhGH doses should be individualized based upon the condition, age, pubertal status, and growth response $[25,26]$.

4.4. Pubertal Dosing. Mauras et al. [9] demonstrated significant improvement in growth response in pubertal children with GHD given higher doses of rhGH $(0.7 \mathrm{mg} / \mathrm{kg} /$ week $)$. Our survey suggests that since the publication and associated U.S. FDA approval of pubertal dosing of Nutropin/Nutropin AQ in GHD patients, many pediatric endocrinologists endorse using higher rhGH doses during puberty, particularly in children not expected to reach expected height. Analysis of the NCGS data documents higher doses used in pubertal patients since the publication and FDA approval of pubertal dosing, but the severity of short stature did not appear to impact this practice. Although these results suggest increased acceptance and use of pubertal dosing, they do not validate it.

4.5. End of Therapy. When determining the right time to stop rhGH therapy, physicians usually consider growth velocity, skeletal maturation, patient comfort with height, severity of GHD, and the presence of other pituitary hormone deficiencies. Survey respondents ranked epiphyseal fusion before patient comfort with height and growth velocity $<2 \mathrm{~cm} / \mathrm{yr}$, although the NCGS data suggest that most patients were stopped before they had stopped growing. Although the data set is small, it suggests that in practice rhGH therapy is discontinued before cessation of growth and that discontinuation may be due to factors other than satisfaction with height, including the adolescent's desire to end therapy.

In children with multiple pituitary hormone deficiencies who have reached adult height, respondents agreed that no retesting was necessary to continue rhGH. This corresponds with recent publications showing that the incidence of adult GHD increases with the number of other pituitary hormone deficiencies [26]. There was no consensus about the timing or methodology (serum IGF-1, GHST) necessary for reevaluation of individuals diagnosed with isolated GHD after achieving adult height.

4.6. Areas in Need of Evidence-Based Guidelines. The combination of the survey responses and their correlation to NCGS data have helped to outline a number of areas of clinical practice variability that require further investigation.

(1) Identification of the best auxologic, imaging, and biochemical profile to diagnose GHD, predict risk of multiple pituitary hormone deficiency, and predict rhGH therapy response.

(2) Establishment of criteria to determine whether growth response to rhGH therapy beyond the first year is adequate and likely to achieve maximal increase in adult height.

(3) Assessment of the utility of monitoring IGF-1 for long-term safety and efficacy of rhGH therapy. 
(4) Identification of the best test and cutoffs for diagnosis of GHD requiring ongoing rhGH therapy after nearadult height is attained.

\section{Conclusions}

The decision-making processes surrounding rhGH treatment are based upon attitudes, beliefs, opinions, and personal experience of the physician. Outside of expert opinion and data gathered for the approval of the current indications for rhGH therapy in children, there are few evidence-based studies to support these processes. The NCGS has been an important resource for safety and efficacy data and can continue to be a data source to provide evidence for development of future consensus guidelines. Our survey, combined with analysis of NCGS data, has outlined several areas that require further clinical study.

\section{Acknowledgments}

This research project was funded by Genentech, Inc. Two of the members of the study group (D. I. Shulman and D. Wyatt) were members of the NCGS Steering Committee. Epi-Q was contracted for its participation in design, performance, and analysis of the study. Members of the study group (B. S. Miller, D. I. Shulman, D. M Wilson, D. Schwartz, M. Kappy and D. Wyatt) were paid as consultants for guidance in designing the study. No funding was provided for study group members for data analysis, data interpretation, or paper preparation. The study board worked independently with EPI-Q during study design and interpretation of the data. Dr. Bakker of Genentech was involved in obtaining and verifying data from NCGS as it related to our survey questions. Because of the funding provided by Genentech as well as the use of NCGS data, the paper was reviewed and approved for publication by Genentech. Editorial comments from Genentech were considered during the preparation of this paper.

\section{References}

[1] "American association of clinical endocrinologists medical guidelines for clinical practice for growth hormone use in adults and children-2003 update," Endocrine Practice, vol. 9, no. 1, pp. 65-76, 2003.

[2] J.-L. André, R. Bourquard, F. Guillemin, M.-J. Krier, and S. Briançon, "Final height in children with chronic renal failure who have not received growth hormone," Pediatric Nephrology, vol. 18, no. 7, pp. 685-691, 2003.

[3] N. J. T. Arends, V. H. Boonstra, and A. C. S. Hokken-Koelega, "Head circumference and body proportions before and during growth hormone treatment in short children who were born small for gestational age," Pediatrics, vol. 114, no. 3, pp. 683690, 2004.

[4] A. F. Attanasio, E. Shavrikova, W. F. Blum et al., "Continued growth hormone $(\mathrm{GH})$ treatment after final height is necessary to complete somatic development in childhood-onset GH-deficient patients," Journal of Clinical Endocrinology and Metabolism, vol. 89, no. 10, pp. 4857-4862, 2004.
[5] R. Ayling, "More guidance on growth hormone deficiency," Journal of Clinical Pathology, vol. 57, no. 2, pp. 123-125, 2004.

[6] A. Badaru and D. M. Wilson, "Alternatives to growth hormone stimulation testing in children," Trends in Endocrinology and Metabolism, vol. 15, no. 6, pp. 252-258, 2004.

[7] A. Bajpai and P. S. N. Menon, "Growth hormone therapy," Indian Journal of Pediatrics, vol. 72, no. 2, pp. 139-144, 2005.

[8] V. K. Bakalov, L. P. Van, J. Baron, J. C. Reynolds, and C. A. Bondy, "Growth hormone therapy and bone mineral density in Turner syndrome," Journal of Clinical Endocrinology and Metabolism, vol. 89, no. 10, pp. 4886-4889, 2004.

[9] M. Bettendorf, H. G. Doerr, B. P. Hauffa et al., "Prevalence of autoantibodies associated with thyroid and celiac disease in Ullrich-Turner syndrome in relation to adult height after growth hormone treatment," Journal of Pediatric Endocrinology and Metabolism, vol. 19, no. 2, pp. 149-154, 2006.

[10] G. Binder, F. Baur, R. Schweizer, and M. B. Ranke, "The d3growth hormone $(\mathrm{GH})$ receptor polymorphism is associated with increased responsiveness to GH in Turner syndrome and short small-for-gestational-age children," Journal of Clinical Endocrinology and Metabolism, vol. 91, no. 2, pp. 659-664, 2006.

[11] G. Binder, M. B. Ranke, and D. D. Martin, "Auxology is a valuable instrument for the clinical diagnosis of SHOX haploinsufficiency in school-age children with unexplained short stature," Journal of Clinical Endocrinology and Metabolism, vol. 88, no. 10, pp. 4891-4896, 2003.

[12] J. Björk, K. Link, and E. M. Erfurth, "The utility of the growth hormone $(\mathrm{GH})$ releasing hormone-arginine test for diagnosing GH deficiency in adults with childhood acute lymphoblastic leukemia treated with cranial irradiation," Journal of Clinical Endocrinology and Metabolism, vol. 90, no. 11, pp. 6048-6054, 2005.

[13] V. H. Boonstra, P. G.H. Mulder, F. H. De Jong, and A. C.S. Hokken-Koelega, "Serum dehydroepiandrosterone sulfate levels and pubarche in short children born small for gestational age before and during growth hormone treatment," Journal of Clinical Endocrinology and Metabolism, vol. 89, no. 2, pp. 712717, 2004.

[14] V. Boonstra, Y. Van Pareren, P. Mulder, and A. HokkenKoelega, "Puberty in growth hormone-treated children born small for gestational age (SGA)," Journal of Clinical Endocrinology and Metabolism, vol. 88, no. 12, pp. 5753-5758, 2003.

[15] A. M. Boot, "Body composition and bone mineral density in adolescents with partial growth hormone deficiency," Journal of Clinical Endocrinology and Metabolism, vol. 88, no. 11, pp. 5099-5100, 2003.

[16] H. R. Boquete, P. G. V. Sobrado, H. L. Fideleff et al., "Evaluation of diagnostic accuracy of insulin-like growth factor (1GF)-1 and IGF-binding protien-3 in growth hormonedeficient children and adults using ROC plot analysis," Journal of Clinical Endocrinology and Metabolism, vol. 88, no. 10, pp. 4702-4708, 2003.

[17] M. Bozzola, F. De Benedetti, M. De Amici et al., "Stimulating effect of growth hormone on cytokine release in children," European Journal of Endocrinology, vol. 149, no. 5, pp. 397401, 2003.

[18] M. Bramnert, M. Segerlantz, E. Laurila, J. R. Daugaard, P. Manhem, and L. Groop, "Growth hormone replacement therapy induces insulin resistance by activating the glucose-fatty acid cycle," Journal of Clinical Endocrinology and Metabolism, vol. 88, no. 4, pp. 1455-1463, 2003. 
[19] C. M. Brownstein, A. C. Mertens, P. A. Mitby et al., "Factors that affect final height and change in height standard deviation scores in survivors of childhood cancer treated with growth hormone: a report from the childhood cancer survivor study," Journal of Clinical Endocrinology and Metabolism, vol. 89, no. 9, pp. 4422-4427, 2004.

[20] J.-C. Carel, "Growth hormone in Turner syndrome: twenty years after, what can we tell our patients?" Journal of Clinical Endocrinology and Metabolism, vol. 90, no. 6, pp. 3793-3794, 2005.

[21] J.-C. Carel, P. Chatelain, P. Rochiccioli, and J.-L. Chaussain, "Improvement in adult height after growth hormone treatment in adolescents with short stature born small for gestational age: results of a randomized controlled study," Journal of Clinical Endocrinology and Metabolism, vol. 88, no. 4, pp. 1587-1593, 2003.

[22] J.-C. Carel, E. Ecosse, I. Bastie-Sigeac et al., "Quality of life determinants in young women with Turner's syndrome after growth hormone treatment: results of the StaTur populationbased cohort study," Journal of Clinical Endocrinology and Metabolism, vol. 90, no. 4, pp. 1992-1997, 2005.

[23] P. V. Carroll, W. M. Drake, K. T. Maher et al., "Comparison of continuation or cessation of growth hormone $(\mathrm{GH})$ therapy on body composition and metabolic status in adolescents with severe GH deficiency at completion of linear growth," Journal of Clinical Endocrinology and Metabolism, vol. 89, no. 8, pp. 3890-3895, 2004.

[24] E. Cecconi, M. Gasperi, F. Bogazzi et al., "Improvement of growth hormone deficiency in patients with primary hyperparathyroidism after parathyroidectomy: results of a prospective study," Journal of Clinical Endocrinology and Metabolism, vol. 89, no. 3, pp. 1213-1216, 2004.

[25] M. Chellakooty, K. Vangsgaard, T. Larsen et al., "A longitudinal study of intrauterine growth and the placental growth hormone (GH)-insulin-like growth factor 1 axis in maternal circulation: association between placental GH and fetal growth," Journal of Clinical Endocrinology and Metabolism, vol. 89, no. 1, pp. 384-391, 2004.

[26] Y. H. Choe, S. Y. Song, K.-H. Paik et al., "Increased density of ghrelin-expressing cells in the gastric fundus and body in Prader-Willi syndrome," Journal of Clinical Endocrinology and Metabolism, vol. 90, no. 9, pp. 5441-5445, 2005. 